Supporting Information for

\title{
Building Multifunctional Meta-Systems via Algorithmic Construction
}

Dayu Zhu ${ }^{1}$, Zhaocheng Liu ${ }^{1}$, Lakshmi Raju ${ }^{1}$, Andrew S. Kim ${ }^{1}$, Wenshan Cai ${ }^{1,2 *}$

${ }^{1}$ School of Electrical and Computer Engineering, Georgia Institute of Technology, Atlanta, Georgia 30332

${ }^{2}$ School of Materials Science and Engineering, Georgia Institute of Technology, Atlanta, Georgia 30332

*Correspondence to: wcai@gatech.edu

\section{Contents:}

1. Structures and training details of the CPPN-GAN

2. Structures and training details of the NN simulator

3. Wave-matrix method for multiple-layer structures

4. Shape tolerance in experiment

5. High-resolution structure of the dual-functional Bessel-Airy beam generator

6. Theoretical model of the all-optical second-order differentiator

7. High-resolution structure of the all-optical second-order differentiator

8. Structures of the 256 unique supercells of the space-polarization-wavelength multiplexed hologram

9. High-resolution output images of the space-polarization-wavelength multiplexed hologram

10. Discussion on the hologram performance and capacity

11. Additional examples of the space-polarization-wavelength multiplexed hologram 


\section{Structures and training details of the CPPN-GAN}

Different from other types of neural network generators, a compositional-pattern generating network (CPPN) takes coordinate $(x, y, r)$ and a latent vector $v$ then outputs an image pixel by pixel. ${ }^{1}$ Thus, a CPPN can generate images with arbitrarily large sizes without losing resolution. Moreover, CPPNs are good at generating artistic images with various styles, which is beneficial for generating nanostructures. A CPPN consists of 8 fully connected layers and the structure is shown in Table S1.

The CPPN is trained in the framework of Generative Adversarial Networks (GAN), ${ }^{2}$ where the CPPN plays a role as a generator, and convolutional neural network $(\mathrm{CNN})$ based discriminator is trained with the generator simultaneously. ${ }^{3}$ The structure of the discriminator is also presented in Table S1. During the training, the discriminator is fed with both the images from the dataset and the images generated by the CPPN, and its duty is to discriminate if the images are real (from the dataset) or fake (generated by the CPPN). As the training proceeds, the CPPN is capable of gradually generating images which are more similar to the real images, and the discriminator also gets better resolving ability. Once the discriminator cannot tell the difference between the real and fake images, we can confidently claim that the CPPN can generate images which are analogous to the ones in the dataset. Mathematically, we denote the real images as $s$, and the fake ones as $\hat{s}$, then the evaluation of the discriminator of the images is denoted as $D_{\omega}(s)$. Since the CPPN takes the coordinates $(x, y, r, v)$ as the input, we can define the CPPN as

$$
\hat{s}=G_{\theta}(x, y, r, z)
$$

with parameters $\theta$. Further, the critics of the discriminator on real and fake images can be respectively written as

$$
\begin{gathered}
l=D_{\omega}(s) \\
\hat{l}=D_{\omega}(\hat{s})=D_{\omega}\left(G_{\theta}(x, y, r, z)\right)
\end{gathered}
$$

with parameters $\omega$. By defining the loss functions

$$
\begin{gathered}
L_{D}(\omega)=\mathbb{E}(l)-\mathbb{E}(\hat{l}) \\
L_{G}(\theta)=-\mathbb{E}(\hat{l})
\end{gathered}
$$

the weights of the CPPN and the discriminator are updated gradually. The training details and the hyperparameters are denoted in Table S1. All codes are written in Python and the deep learning algorithm is implemented in PyTorch 1.0. The implementation, training, and execution of our DL framework are conducted on an Ubuntu system with an Nvidia Quadro P5000 GPU, an Intel Xeon E5-1650 CPU and 32 GB memory. 


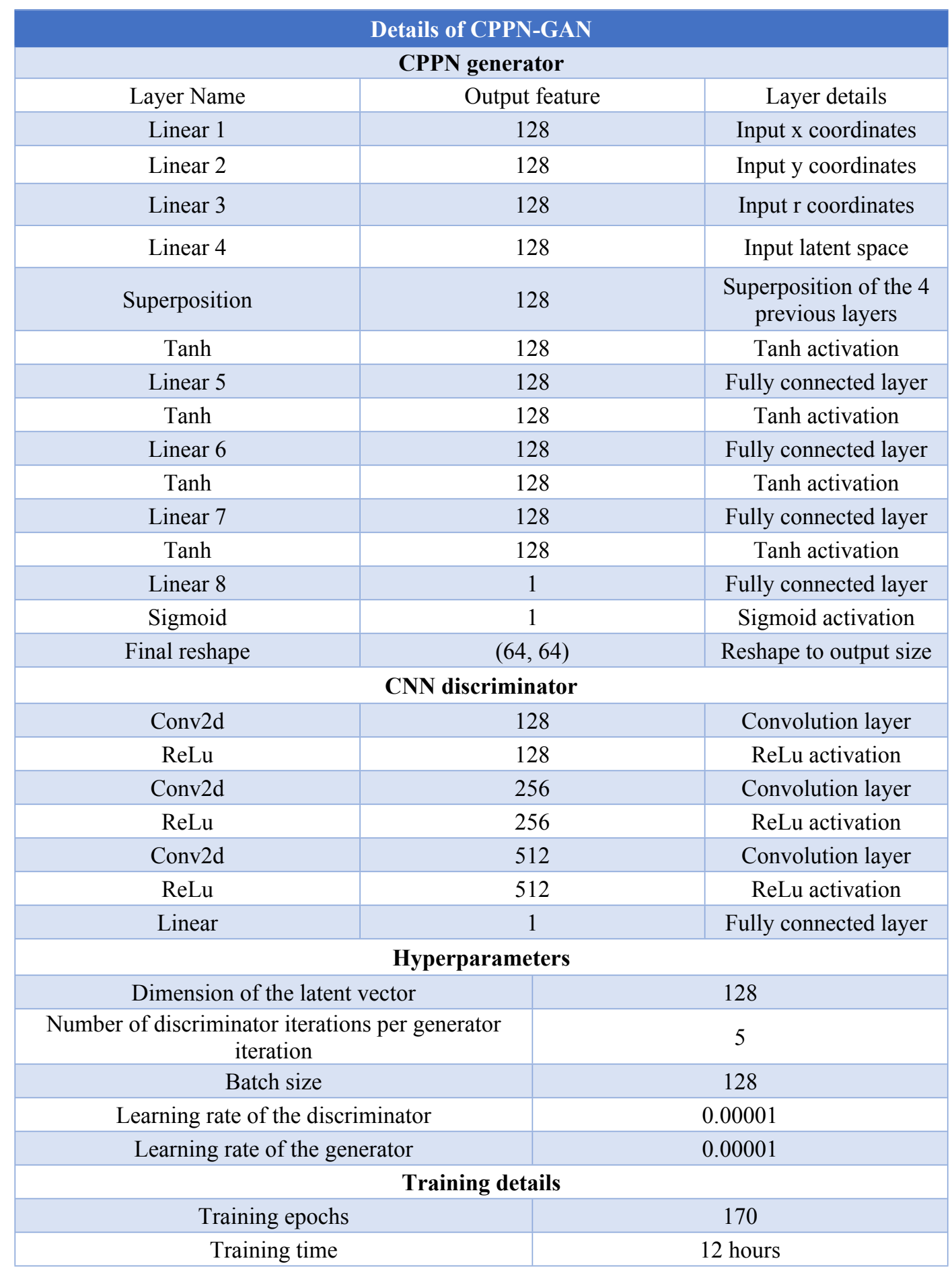

Table S1. Structures and training details of the CPPN-GAN. 


\section{Structures and training details of the NN simulator}

A neural network $(\mathrm{NN})$ simulator computes the corresponding optical responses of the patterns which are produced by the generator. Compared with commercial software, such as COMSOL, the NN simulator is much more efficient and able to processes hundreds of computations in seconds and provide satisfactory accuracy. The NN simulator is constructed using a popular CNN architecture, ResNet ${ }^{4}$, which applies the skip-connection architecture to allow the training of a deep NN to be feasible. Here we use ResNet18, which is deep enough for the complexity of our tasks and is not too deep to overfit our dataset. The NN simulator takes 64 by 64 pixelated images as the input and the complex transmission and reflection spectra $(170 \mathrm{THz}$ to $600 \mathrm{THz}$, i.e. 500 $\mathrm{nm}$ to $1765 \mathrm{~nm}$ in free pace) as the output. Each image represents the two-dimensional nanostructure of a unit cell, with a periodicity of $320 \mathrm{~nm}$ and the nanostructure thickness of $40 \mathrm{~nm}$. To be reasonable for multilayer designs, we assume all nanostructures are gold and embedded in a polymer with a refractive index of 1.57. The spectra shows the four-component complex transmission (reflection) responses of the unit cell when a plane wave with $x$ - or $y$-polarization is incident, which can be denoted as $T_{x x}, T_{x y}, T_{y x}, T_{y y}\left(R_{x x}, R_{x y}, R_{y x}\right.$, and $\left.R_{y y}\right)$. The spectra cover every $10 \mathrm{THz}$ from $170 \mathrm{THz}$ to $600 \mathrm{THz}$, which consist of 44 wavelength points within the range. Thus, we have adapted the last layer of ResNet18 to make it generate both the real and imaginary parts of the transmission or reflection responses. Two NN simulators with the same architecture are trained to yield the transmission and reflection spectra, respectively, and each output dimension is $4 \times 44=176$. The detailed architecture of the NN simulator is shown in Table. $\mathbf{S 2}$.

Training a deep neural network requires a huge amount of data, and such a dataset in photonics is not readily available. The researchers need to generate their own dataset using commercial simulation software, which is cumbersome and limits the usefulness of deep learning in photonics. Here we claim that applying transfer learning mitigates the dilemma effectively. We only need to generate a large dataset once, and every time we want to use the NN simulator in other tasks, only a small dataset is needed to transfer the knowledge from the large dataset to the new small dataset. Here, although the NN simulator is a very deep NN, it is trained with a small dataset of only 2,000 image-spectra pairs. We avoid simulating the spectra of tons of nanostructures to generate the training dataset by applying transfer learning to reduce the data requirement. In our previous research, we have simulated 16,000 image-spectra data pairs of unit cells of the same periodicity and height, where the gold structure is deposited on glass and exposed in air. Although the material configurations are different, transfer learning can find the relationships. We first use the previous data (air-glass-gold configuration) to train our simulator and get $98 \%$ accuracy. Then while

freezing all the layers of the simulator except for the last layer, we continue training the simulator with only 2,000 new data points (polymer-gold configuration) and get $96 \%$ accuracy. The high efficacy of the simulator suggests transferring learning successfully transfers the knowledge from the air-glass environment to the polymer-embedded environment. It also foreshadows that our framework can be readily transferred into other systems of materials or sizes. The training details are presented in Table S2. 


\begin{tabular}{|c|c|c|}
\hline \multicolumn{3}{|c|}{ Details of the NN Simulator } \\
\hline \multicolumn{3}{|c|}{ ResNet simulator } \\
\hline Layer Name & Output size (if Batch size = 64) & Layer details \\
\hline conv1 & $64 \times 64 \times 64$ & With BatchNorm \\
\hline Buildblock1 & $64 \times 64 \times 64$ & $\begin{array}{c}{\left[\begin{array}{c}3 \times 3,64 \\
3 \times 3,64\end{array}\right] \times 2} \\
\text { stride } 2\end{array}$ \\
\hline Buildblock2 & $128 \times 32 \times 32$ & $\begin{array}{c}{\left[\begin{array}{c}3 \times 3,128 \\
3 \times 3,128\end{array}\right] \times 2} \\
\text { stride } 2\end{array}$ \\
\hline Buildblock3 & $256 \times 16 \times 16$ & $\begin{array}{c}{\left[\begin{array}{c}3 \times 3,256 \\
3 \times 3,256\end{array}\right] \times 2} \\
\text { stride } 2\end{array}$ \\
\hline Buildblock4 & $128 \times 8 \times 8$ & $\begin{array}{c}{\left[\begin{array}{l}3 \times 3,128 \\
3 \times 3,128\end{array}\right] \times 2} \\
\text { stride } 2\end{array}$ \\
\hline Average pool & $128 \times 2 \times 2$ & $4 \times 4$ average pool \\
\hline Linear 1 & 128 & $\begin{array}{l}\text { Fully connected } \\
\text { layer }\end{array}$ \\
\hline ReLu & 128 & ReLu activation \\
\hline Linear 2 & $176 \times 2$ & $\begin{array}{l}\text { Fully connected } \\
\text { layer }\end{array}$ \\
\hline \multicolumn{3}{|c|}{ Hyperparameters } \\
\hline \multicolumn{2}{|c|}{ Learning rate of the training on the big dataset } & 0.00005 \\
\hline \multicolumn{2}{|c|}{ Learning rate of the transfer learning on the small dataset } & 0.00001 \\
\hline \multicolumn{2}{|c|}{ Batch size } & 64 \\
\hline \multicolumn{3}{|c|}{ Training details (big dataset) } \\
\hline \multicolumn{2}{|c|}{ Training epochs } & 200 \\
\hline \multicolumn{2}{|c|}{ Training time } & 20 hours \\
\hline \multicolumn{3}{|c|}{ Training details (transfer learning) } \\
\hline \multirow{2}{*}{\multicolumn{2}{|c|}{$\begin{array}{l}\text { Training epochs } \\
\text { Training time }\end{array}$}} & 50 \\
\hline & & 3 hours \\
\hline
\end{tabular}

Table S2. Structure and training details of the NN simulator.

\section{Wave-matrix method for multiple-layer structures}

We use wave matrices to formulate the properties of layered structures ${ }^{5}$. In principle, we can generalize every layer of a metasurface as a thin interface between dielectrics. The transmission and reflection properties of an interface can be represented by an $S$ Matrix as

$$
\left(\begin{array}{c}
E_{1}^{-} \\
E_{2}^{+}
\end{array}\right)=\left(\begin{array}{ll}
S_{11} & S_{12} \\
S_{21} & S_{22}
\end{array}\right)\left(\begin{array}{c}
E_{1}^{+} \\
E_{2}^{-}
\end{array}\right),
$$

where $E_{i}^{+}$and $E_{i}^{-}$represent the forward and backward waves in region $I, S_{i j}$ are $2 \times 2$ blocks of the $S$ matrix, and the $S$ Matrix describes the properties of the interface between region 1 and region 2 . The relationship between the $S$ Matrix and the Wave Matrix (represented by $M$ ) of the interface is 


$$
M_{\text {inter }}=\left(\begin{array}{cc}
I & 0 \\
S_{11} & S_{12}
\end{array}\right)\left(\begin{array}{cc}
S_{21} & S_{22} \\
0 & I
\end{array}\right)^{-1},
$$

where $I$ and 0 are the $2 \times 2$ identity matrix and the zero matrix, respectively. Furthermore, the dielectric spacer between two interfaces, if isotropic, has the $4 \times 4 M$ matrix

$$
M_{\text {delay }}=\left[\begin{array}{cccc}
e^{j \theta} & 0 & 0 & 0 \\
0 & e^{j \theta} & 0 & 0 \\
0 & 0 & e^{-j \theta} & 0 \\
0 & 0 & 0 & e^{-j \theta}
\end{array}\right],
$$

where $\theta$ is the phase delay of the wave when propagating through the dielectric spacer. Thus, the overall performance of the meta-optic system can be formulated as

$$
\left(\begin{array}{l}
E_{1}^{+} \\
E_{1}^{-}
\end{array}\right)=M_{\text {inter }}^{(1)} M_{\text {delay }}^{(2)} M_{\text {inter } \ldots}^{(2)} M_{\text {delay }}^{(n-1)} M_{\text {inter }}^{(n-1)}\left(\begin{array}{c}
E_{n}^{+} \\
E_{n}^{-}
\end{array}\right)
$$

if it has $\mathrm{n}$ regions in total.

\section{Shape tolerance in experiment}

The patterns of the designed metasurface can sometimes be too complicated for fabrication. Fortunately, this issue can be settled by selection and smoothing in practical use. During the design process of a unit cell, we first run the framework several times to generate multiple candidates. Then we select the most practical one for fabrication. Besides, we can apply a denoising algorithm and a Fourier transform filter (FTF) to smooth the patterns ${ }^{6}$. The denoising algorithm will erase isolated small dots on the image as well as fill some small holes. Keeping a relative low filtering order, the FTF will smooth the superfine features of the patterns and only leave the major contours. Since the loss of tiny features will not affect the far field performance of the unit cell, it is safe to use denoising algorithm and FTF if the near field properties are not of interest. Figure S1(a) illustrates how the image processing procedure works and Figure S1(b) presents some examples of the patterns processed by the procedure. As an experimental example, Figure S1(c) shows the designed structures and the scanning electron microscope (SEM) image of a fabricated metasurface. The metasurface consists of 8 unique unit cells, each with size of $320 \mathrm{~nm}$ and a 40 $\mathrm{nm}$-thick gold structure sited on glass substrate. The device is designed to convert $x$-polarized incident light into $y$-polarized output. Although the fabricated device shows some discrepancy from the designed patterns, it still fulfills the design objective successfully. This experimental result justifies that trivial features of the structure will not influence the overall far field performance 7 . In addition, the fabrication of multilayered structures in optical wavelengths is very demanding, while much easier for microwave devices. This work majorly focusses on the capacity of the design framework and the potential of meta-optic systems, experimental demonstration is left for future research. 


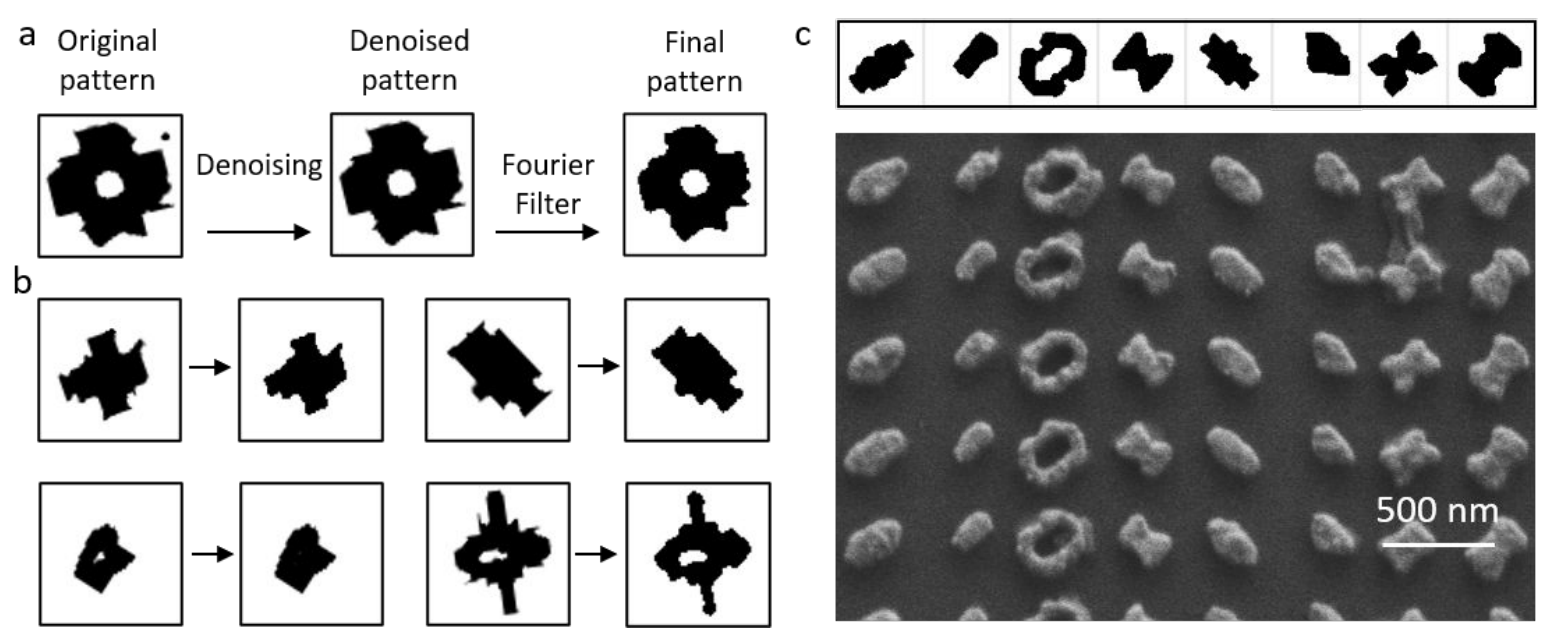

Figure S1. Fine features of structures will not affect the performance. (a) The procedure to generate the fabrication-friendly structures. The denoising algorithm is first applied to wipe out isolated small dots or fill small holes. Then the Fourier transform filter is applied to smooth the sharp edges and corners of the pattern. (b) Four examples illustrate the effects of the image processing procedure. (c) A designed metasurface and the SEM image of the fabricated sample. The metasurface consists of 8 unique unit cells and the function is to convert $x$-polarized incident light into $y$-polarized output. The discrepancy between the target structure and the fabricated structure does not influence the performance of the metasurface.

\section{High-resolution structure of the dual-functional Bessel-Airy beam generator}

In our main text we have presented a dual-functional Bessel-Airy beam generator. The beam generator has three layers, each layer consisting of 25 by 25 unit cells. Figure S2 presents the high-resolution structures of the beam generator, from the top layer to the bottom layer, respectively. This figure is across three pages. As we have discussed in the Shape tolerance in experiment section, even though some unit cells are too complicated to fabricate, we can perform denoising and FTF processes to obtain the fabrication-friendly structures without sacrificing the performance. 


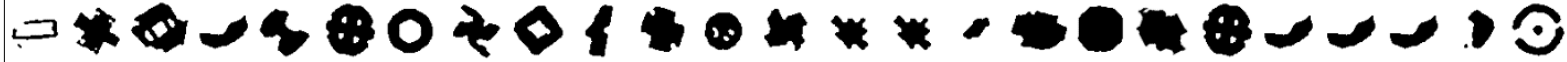

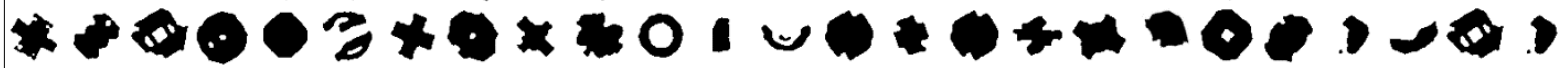
0.109010 .1 .1 .10100100

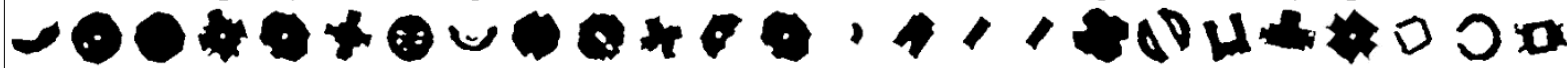

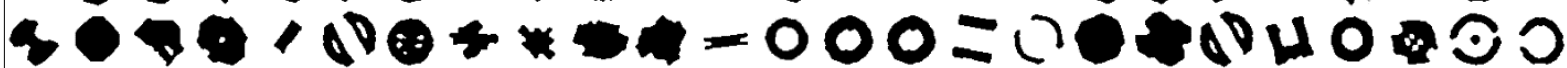

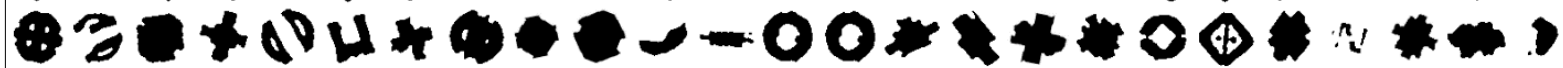

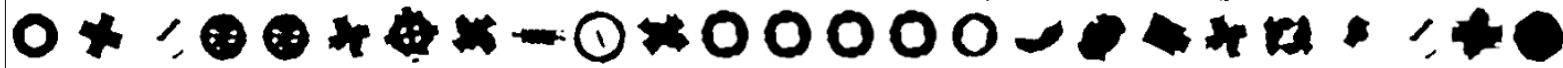

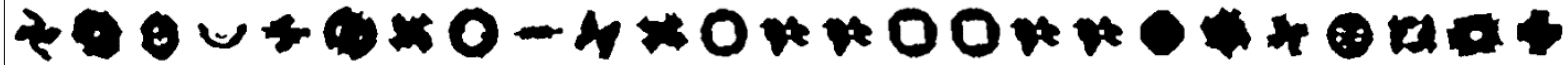
- * * * - - - - - $400 * 4+1001$

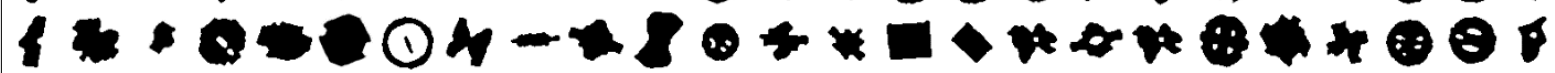

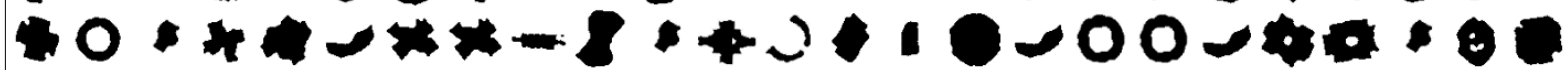

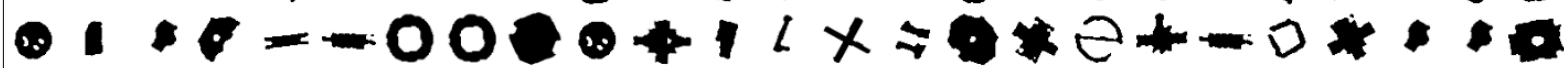
$4 \cup 0000 *+j L \cdot 11 \cdot 14-40-1 \cdot 10$

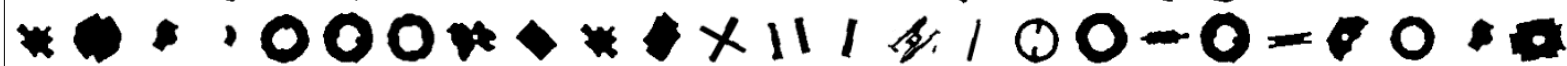
* 10*000च1

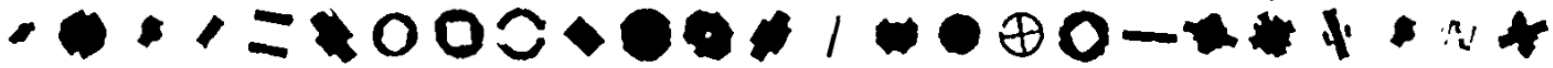

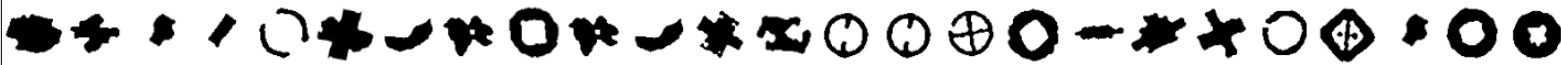

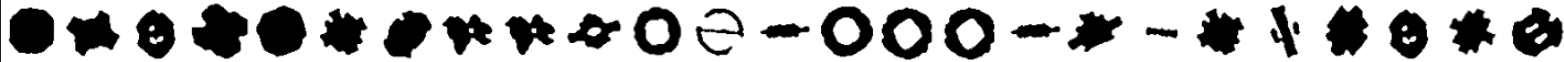
- 1000004*0+4--1*-100.000

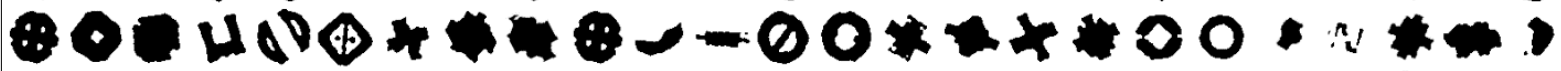

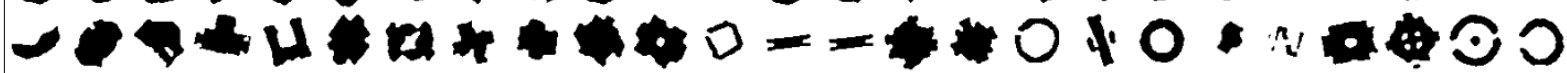

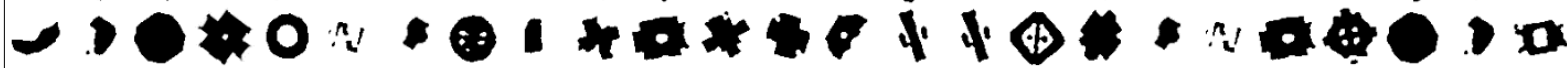

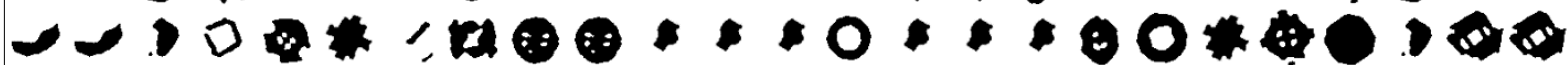

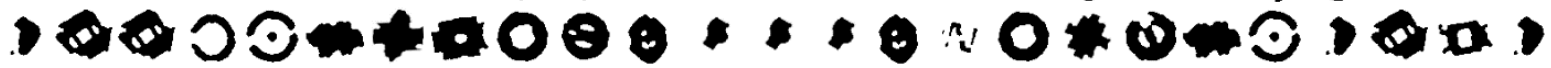

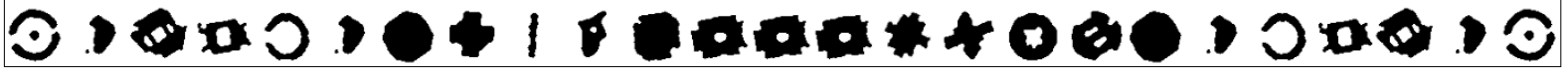




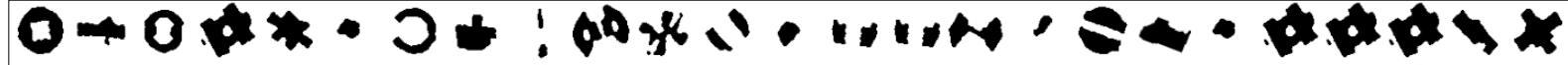

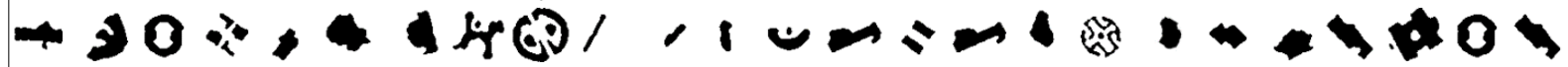

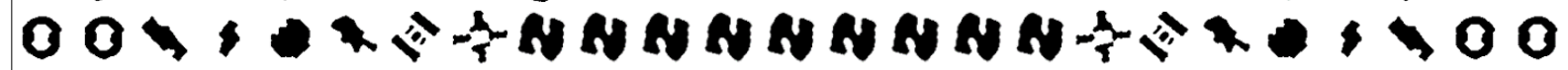

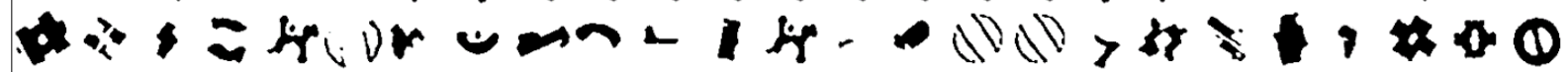

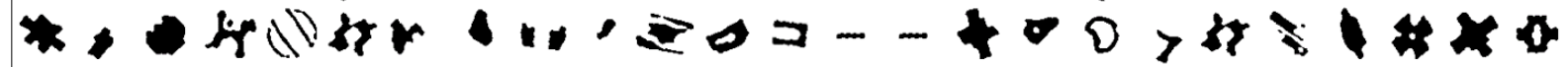

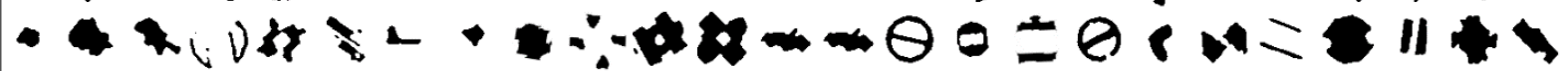
o ANr

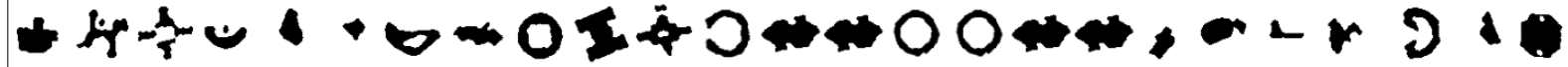

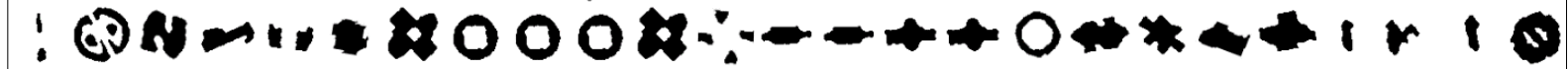

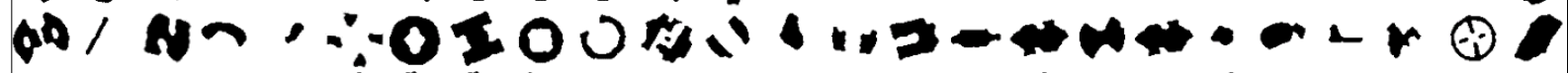

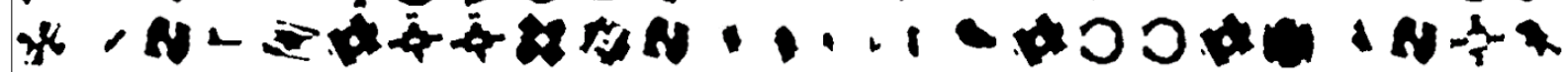

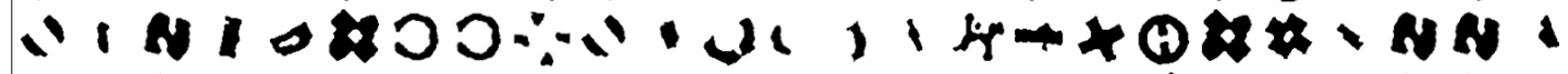

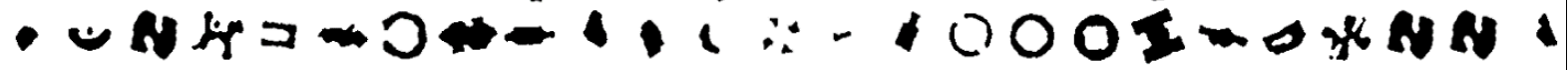

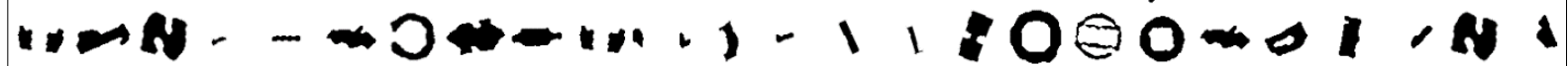
\#N- $-\theta 00+211+1) \theta 0-0+1=N-11$

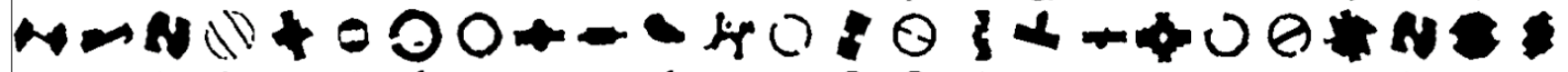

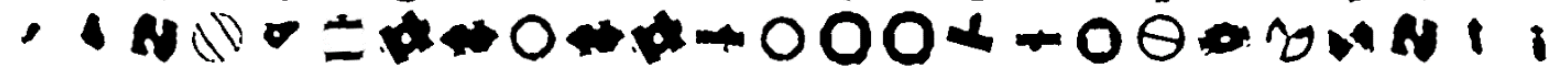
$-4-0 \theta+40+0 \theta-0 \theta-\theta+-4=-110$

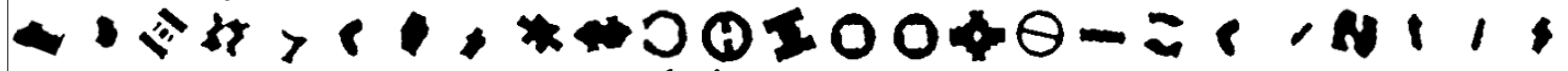

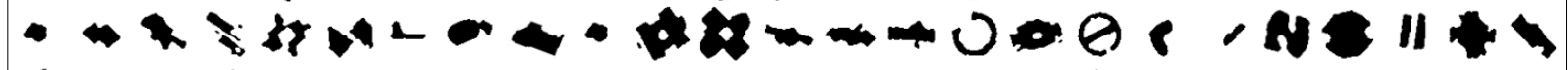

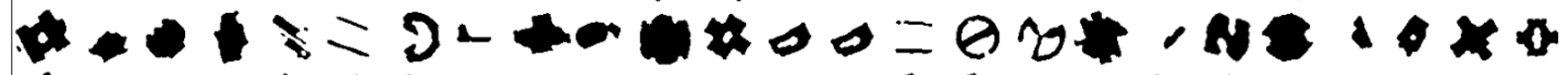

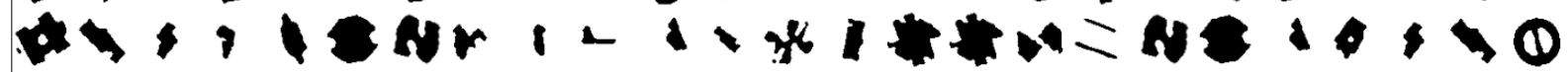

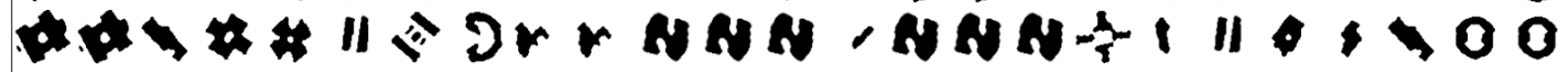

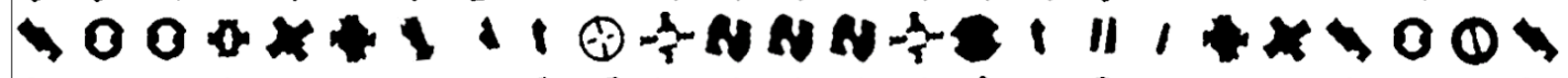

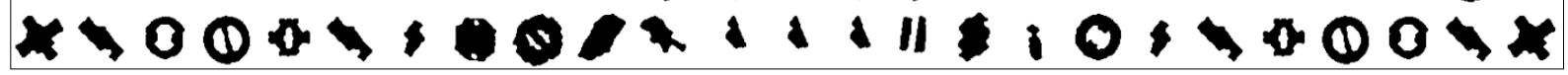




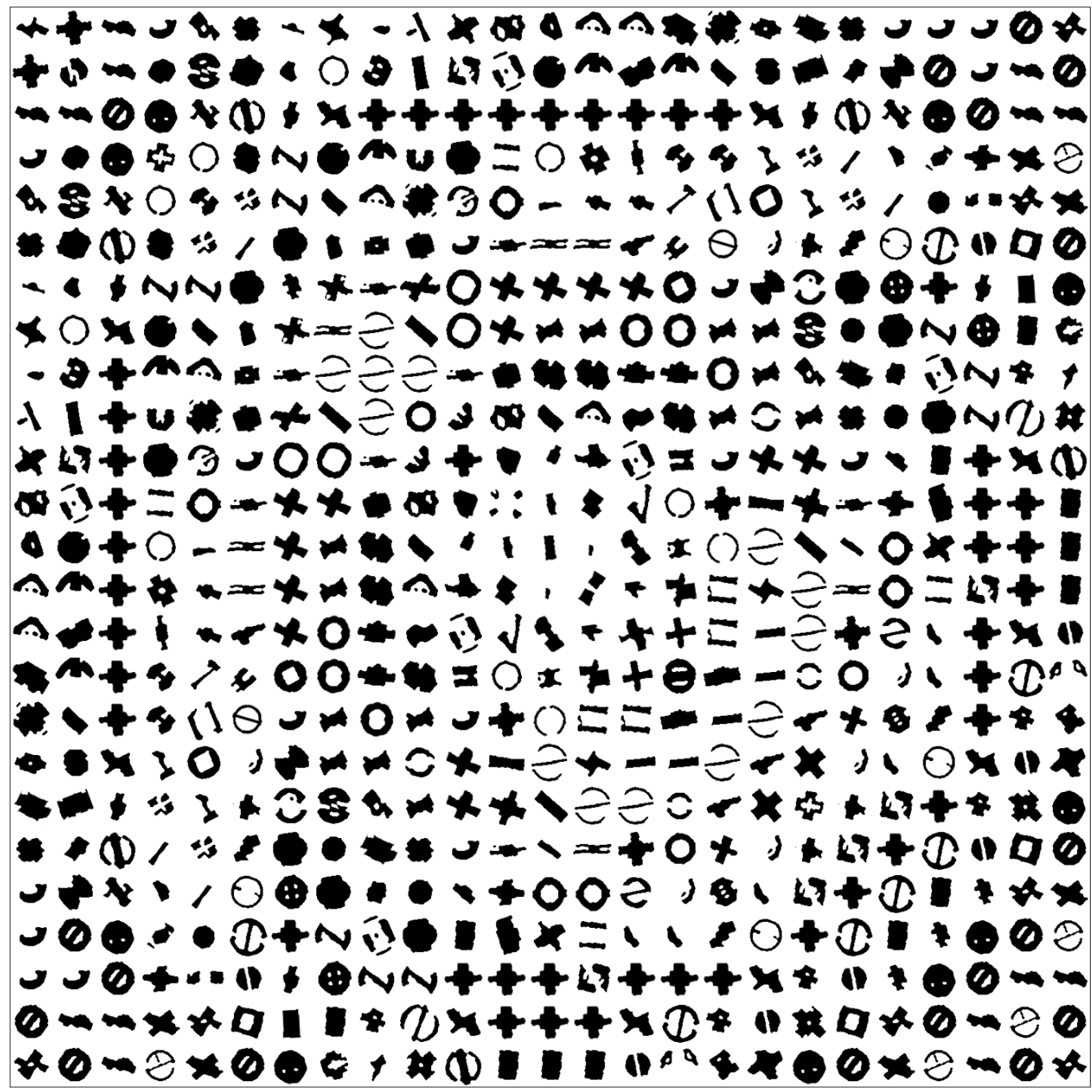

Figure S2. High-resolution structure of the dual-functional Bessel-Airy beam generator. The beam generator has three layers, each layer consists of $25 \times 25$ unit cells. This figure spans three pages and shows the structures from Layer 1 to Layer 3 sequentially. 


\section{Theoretical model of the all-optical second-order differentiator}

The theoretical background of the all-optical second-order differentiator is based on the Green's function of a linear operator ${ }^{8}$. Without loss of generality, we assume a linear operator $L$ acting over the $x$ direction, which is denoted as $L=L(x)$. The equation has the general form

$$
\operatorname{Lu}(x)=f(x)
$$

where $u(x)$ is the expected solution of the equation, while $f(x)$ is an $x$-variant term. Then there will be at least one Green's function, $G(x, s)$, which is the solution to

$$
L G(x, s)=\delta(x-s),
$$

where $\delta(x)$ is the Dirac delta function over $x$. Thus, the Green's function can be regarded as the kernel of the inverse operator of $L$. Combining equations (10 and 11) and integrating $L G(x, s) f(s)$ (10) over $s$ leads to

$$
L\left(\int G(x, s) f(s) d s\right)=\int L G(x, s) f(s) d s=\int \delta(x-s) f(s) d s=f(x),
$$

which means

$$
u(x)=\int G(x, s) f(s) d s
$$

is a solution to equation (10). Specially, if $L$ is invariant along $x$, the Green's function can be expressed as $G(x, s)=G(x-s)$. As a result, $u(x)$ can be rewritten as the convolution form

$$
u(x)=\int G(x-s) f(s) d s=G(x) * f(x) .
$$

In this sense, $f(x)$ is the input of the device for all-optical computation and $u(x)$ is the output (solution).

In the case of a second-order differentiator, the differential equation can be expressed as

$$
u(x)=\frac{\partial^{2} f(x)}{\partial x^{2}}
$$

So the Green's function of $L$ satisfies

$$
L G(x, s)=\iint G(x-s) d x d x=\delta(x-s)
$$

And we derive the Green's function

$$
G(x, s)=G(x-s)=\delta^{\prime \prime}(x-s),
$$


which is the second derivative of the Dirac delta function. Since the perfect delta function does not exist in the real world, we use a Gaussian function with a small half bandwidth to approach it. The transmittance in $\mathrm{k}$-space is proportional to the Fourier transform of $\delta^{\prime \prime}(x-s)$, i.e.

$$
T\left(k_{x}\right) \propto \mathcal{F}(G(x, s))=-k_{x}{ }^{2} .
$$

\section{High-resolution structure of the all-optical second-order differentiator}

In our main text we have presented an all-optical second-order differentiator. The device possesses three layers, each layer consisting of 25 unit cells along the $x$-direction and periodic along the $y$ direction. Figure S3 presents the high-resolution structures of the second-order differentiator, from the top layer (layer 1) to the bottom layer (layer 3), respectively. The incidence is on the layer 1 side and the output is on the layer 3 side. As we have discussed in the Shape tolerance in experiment section, if some structures are hard for fabrication, we can perform denoising and FTF to obtain the fabrication-friendly structures without sacrificing the performance. If we design a differentiator with more supercells, the performance will be improved as well. 


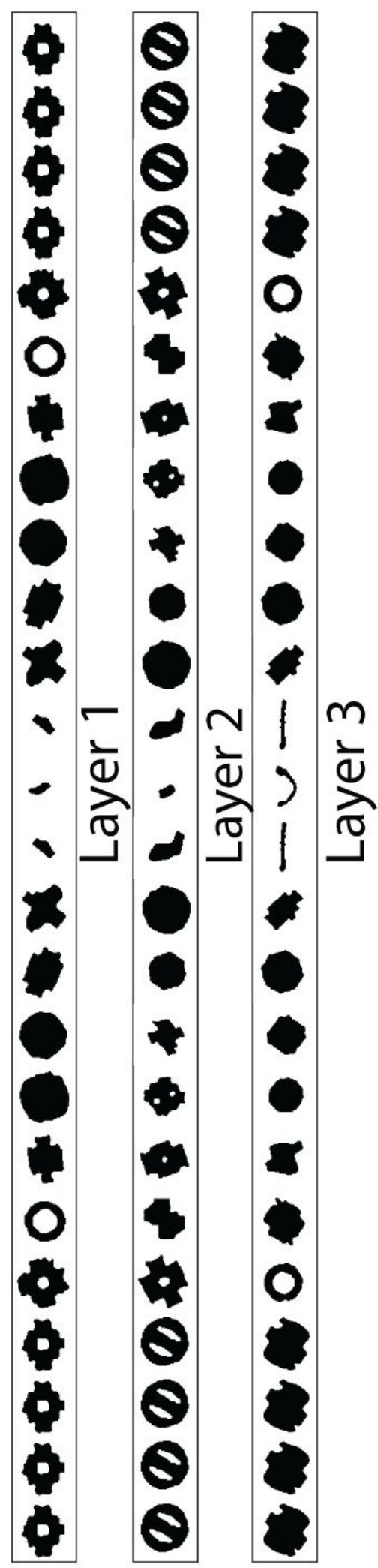

Figure S3. High-resolution structure of the all-optical second-order differentiator. 


\section{Structures of all the 256 unique supercells for space-polarization-wavelength multiplexed holograms}

In our main text we have presented a space-polarization-wavelength multiplexed hologram. The hologram is made up of 2000 by 2000 supercells, and there are 256 unique supercells among all the $4 \times 10^{6}$ supercells. Each supercell has three layers, and each layer is separated by a $200 \mathrm{~nm}$ polymer spacer. The detailed structures of all 256 supercells are presented in Figure S4. The three patterns of each supercell, from the left to the right, are the top layer to the bottom layer. The target responses for $x$ and $y$-polarized incidence at $562 \mathrm{~nm}\left(\lambda_{1}\right)$ and $659 \mathrm{~nm}\left(\lambda_{2}\right)$ of the supercell are denoted as $E_{\lambda_{1} x}, E_{\lambda_{1} y}, E_{\lambda_{2} x}, E_{\lambda_{2} y}$ below each supercell. During the design process, we run the deep learning design framework 10 times to get 10 candidates of each unique super cell. Then we simulate the responses of all 2560 supercells in COMSOL and select the best design for each super cell from the candidate pool. Thus, some candidates may fit for more than one objective within the error range (15\%). Although some simulated responses of the supercells do not perfectly fit their targeted ones, the central limit theorem (CTL) guarantees that the overall performance of the hologram deviates not too far from the design objective. If we increase the repetition rate from 10 to a larger value, we will attain supercells which better fulfill the design objectives. 


\section{$\because \because: \bigcirc$ \\ $0,0,0,0$ \\ $\rightarrow \infty$}

$0,0,0,0.75 \oplus \mathrm{p} / 3$

$+8 x$

0, $0,0.75 @-p i, 0.759-p i / 3$

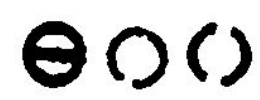

$0,0,0.75 @-p i / 3,0.75 @-p i$

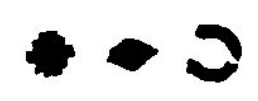

$0,0,0.75 \otimes \mathrm{pi} / 3,0$

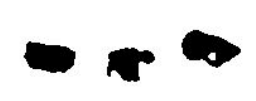

$0,0,0.75 @ \mathrm{pi} / 3,0.75 @ \mathrm{pi} / 3$

\section{OO II}

$0,0.75 @-p i, 0,0.759-p i / 3$

$$
12
$$

$0,0.75 @-p i, 0.75 @-p i, 0.75 @-p i$

\section{ง}

$0,0.750-p i, 0.75 @-p i / 3,0$

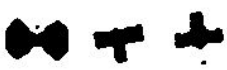

$0,0.75 @-p i, 0.75 @-p i / 3,0.75 @ p i / 3$

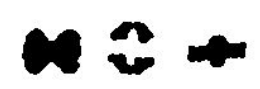

0, $0.75 @-p i, 0.75 @ p i / 3,0.75 @-p i / 3$

\section{o e o}

$0,0.75 @-p i / 3,0,0.75 @-p i$

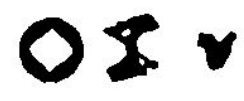

0, $0.75 @-p i / 3,0.75 @-p i, 0$

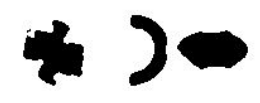

0, 0.75@-pi/3, 0.75@-pi, 0.75@pi/3

\section{$0 \theta ?$}

$0,0,0,0.75 \Theta-p i$

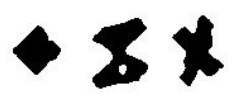

$0,0,0.75 @-p i, 0$

\section{$\rightarrow 52$}

0, 0, $0.75 @-p i, 0.759 p i / 3$

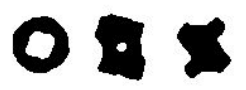

$0,0,0.75 @-\mathrm{pi} / 3,0.75 @-\mathrm{pi} / 3$

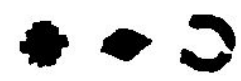

$0,0,0.75 @ \mathrm{pi} / 3,0.75 @-p i$

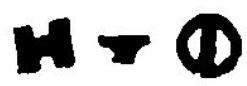

0, 0.75@-pi, 0, o

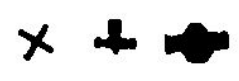

$0,0.750-p i, 0,0.759 \mathrm{pi} / 3$

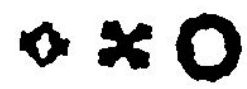

0, $0.75 @-p i, 0.75 @-p i, 0.75 @-p i / 3$

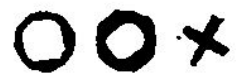

0, $0.75 @-p i, 0.75 @-p i / 3,0.75 @-p i$

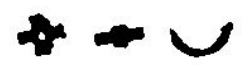

0, 0.75@-pi, 0.75@pi/3,0

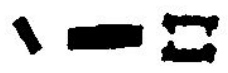

$0,0.75 @-p i, 0.75 @ \mathrm{pi} / 3,0.75 @ \mathrm{pi} / 3$

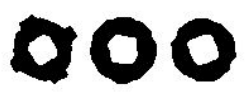

$0,0.75 @-p i / 3,0,0.75 @-p i / 3$

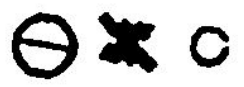

$0,0.750-p i / 3,0.750-p i, 0.75 @-p i$

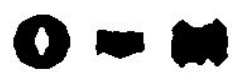

$0,0.750-p i / 3,0.750-p i / 3,0$
$02 \pi$

$0,0,0,0.75 @-\mathrm{pi} / 3$

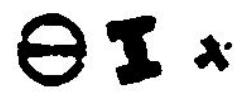

$0,0,0.75 @-p i, 0.759-p i$

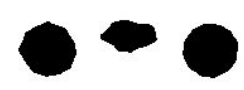

$0,0,0.75 @-\mathrm{pi} / 3,0$

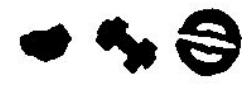

$0,0,0.75 @-p i / 3,0.75 @ p i / 3$

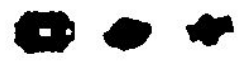

$0,0,0.75 @ \mathrm{pi} / 3,0.75 @-\mathrm{pi} / 3$

$00 \cdot 1$

$0,0.75 @-p i, 0,0.759-p i$

v. 520

$0,0.75 @-p i, 0.75 @-p i, 0$

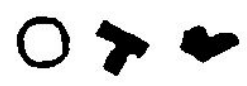

0, $0.75 @-p i, 0.75 @-p i, 0.75 @ p i / 3$

OO II

$0,0.75 @-p i, 0.75 @-p i / 3,0.75 @-p i / 3$

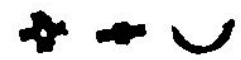

$0,0.75 @-p i, 0.75 @ p i / 3,0.75 @-p i$

$0=0$

$0,0.750-\mathrm{pi} / 3,0,0$

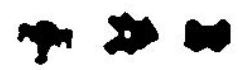

$0,0.75 @-\mathrm{pi} / 3,0,0.75 @ \mathrm{pi} / 3$

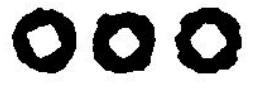

$0,0.75 @-p i / 3,0.75 @-p i, 0.75 @-p i / 3$

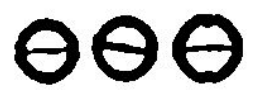

$0,0.75 @-p i / 3,0.75 @-p i / 3,0.75 @-p i$ 


\section{0}

$0,0.75 @-p i / 3,0.75 @-p i / 3,0.75 @-p i / 3$

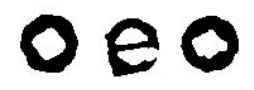

$0,0.75 @-p i / 3,0.75 @ p i / 3,0.75 @-p i$

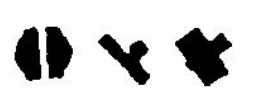

$0,0.75 @ p i / 3,0,0$

\section{$x<$}

$0,0.75 @ p i / 3,0,0.75 @ p i / 3$

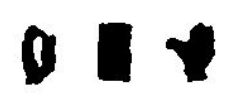

$0,0.75 @ \mathrm{pi} / 3,0.75 @-\mathrm{pi}, 0.75 @-\mathrm{pi} / 3$

\section{$+1$}

$0,0.75 @ \mathrm{pi} / 3,0.75 @-\mathrm{pi} / 3,0.75 @-\mathrm{pi}$

\section{$1+0$}

$0,0.75 @ \mathrm{pi} / 3,0.75 @ \mathrm{pi} / 3,0$

$$
<\Leftrightarrow
$$

$0,0.75 @ \mathrm{pi} / 3,0.75 @ \mathrm{pi} / 3,0.75 @ \mathrm{pi} / 3$

\section{OH \\ $0.75 @-p i, \quad 0,0,0.75 @-p i / 3$

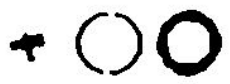 \\ $0.75 @-p i, \quad 0,0.75 @-p i, \quad 0.75 @-p i$ \\ N 0 \\ $0.75 @-p i, 0,0.75 @-p i / 3,0$

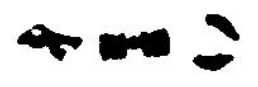 \\ $0.75 @-p i, 0,0.75 @-p i / 3,0.75 @ p i / 3$

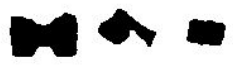

$0.75 @-p i, \quad 0,0.75 @ p i / 3,0.75 @-p i / 3$

\section{nゆ)}

0.75@-pi, 0.75@-pi, 0, 0.75@-pi

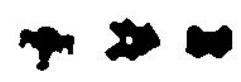

$0,0.75 @-p i / 3,0.75 @-p i / 3,0.75 @ p i / 3$

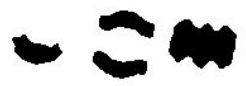

$0,0.75 @-p i / 3,0.75 @ p i / 3,0.75 @-p i / 3$

$$
>1
$$

$0,0.75 @ p i / 3,0,0.75 @-p i$

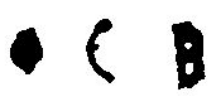

$0,0.75 @ \mathrm{pi} / 3,0.75 @-\mathrm{pi}, 0$

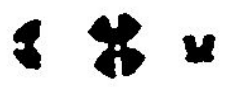

$0,0.75 @ \mathrm{pi} / 3,0.75 @-\mathrm{pi}, 0.75 @ \mathrm{pi} / 3$

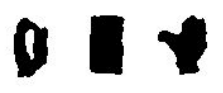

$0,0.75 @ \mathrm{pi} / 3,0.75 @-\mathrm{pi} / 3,0.75 @-\mathrm{pi} / 3$

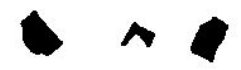

$0,0.75 @ \mathrm{pi} / 3,0.75 @ \mathrm{pi} / 3,0.75 @-\mathrm{pi}$

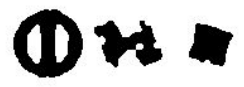

$0.75 @-p i, 0,0,0$

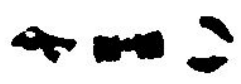

$0.75 @-p i, 0,0,0.75 @ p i / 3$

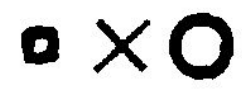

$0.75 @-p i, \quad 0,0.75 @-p i, \quad 0.75 @-p i / 3$

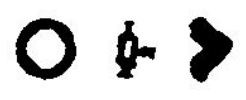

$0.75 @-p i, 0,0.75 @-p i / 3,0.75 @-p i$

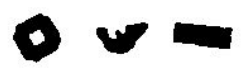

$0.75 @-p i, 0,0.75 @ p i / 3,0$

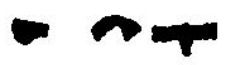

$0.75 @-p i, \quad 0,0.75 @ p i / 3,0.75 @ p i / 3$

\section{$\mathbb{D Q} \times$}

$0.75 @-p i, 0.75 @-p i, \quad 0,0.75 @-p i / 3$ $x=\dot{x}$

$0,0.75 @-p i / 3,0.75 @ p i / 3,0$

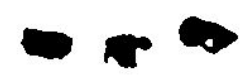

$0,0.75 @-\mathrm{pi} / 3,0.75 @ \mathrm{pi} / 3,0.75 @ \mathrm{pi} / 3$

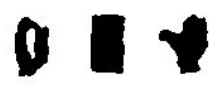

$0,0.75 @ \mathrm{pi} / 3,0,0.75 @-\mathrm{pi} / 3$

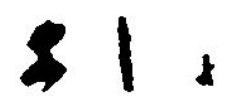

$0,0.75 @ p i / 3,0.75 @-p i, 0.75 @-p i$

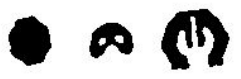

$0,0.75 @ p i / 3,0.75 @-p i / 3,0$

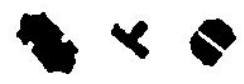

$0,0.75 @ \mathrm{pi} / 3,0.75 @-\mathrm{pi} / 3,0.75 @ \mathrm{pi} / 3$

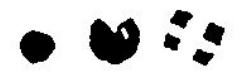

$0,0.75 @ \mathrm{pi} / 3,0.75 @ \mathrm{pi} / 3,0.75 @-\mathrm{pi} / 3$

() 00 $0.75 @-p i, 0,0,0.75 @-p i$

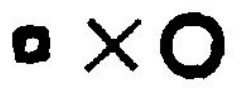

$0.75 @-p i, 0,0.75 @-p i, 0$

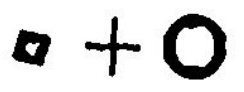

$0.75 @-p i, \quad 0, \quad 0.75 @-p i, \quad 0.75 @ p i / 3$

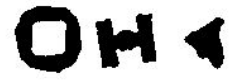

$0.75 @-p i, 0,0.75 @-p i / 3,0.75 @-p i / 3$

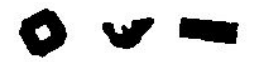

$0.75 @-p i, 0,0.75 @ \mathrm{pi} / 3,0.75 @-\mathrm{pi}$

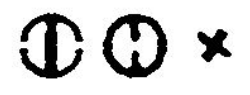

$0.75 @-p i, 0.75 @-p i, 0,0$

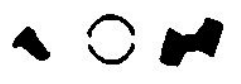

$0.75 @-p i, \quad 0.75 @-p i, \quad 0,0.75 @ p i / 3$ 


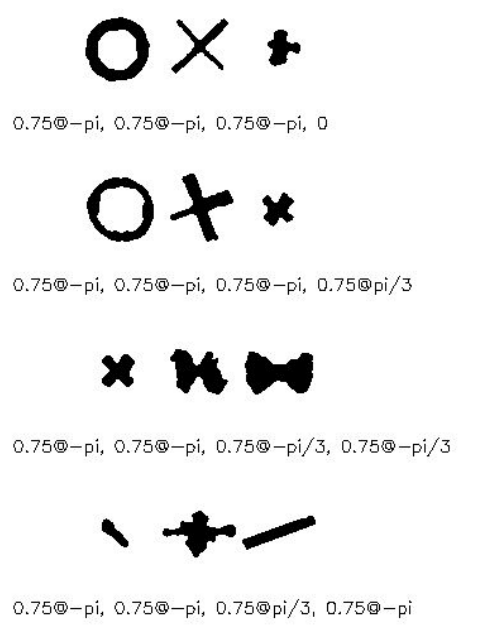

(1)

$0.75 @-p i, \quad 0.75 @-p i / 3,0,0$

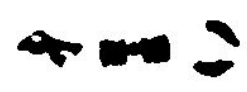

$0.75 @-p i, 0.75 @-p i / 3,0,0.75 @ p i / 3$

\section{$0 \times 0$}

$0.75 @-p i, 0.75 @-p i / 3,0.75 @-p i, 0.75 @-p i / 3$

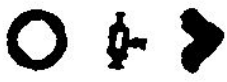

$0.75 \Theta-p i, \quad 0.75 \Theta-p i / 3,0.75 \Theta-p i / 3,0.75 \Theta-p i$

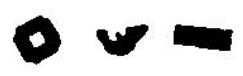

$0.75 @-p i, 0.75 @-p i / 3,0.75 @ p i / 3,0$

\section{$\operatorname{mot} \theta$}

$0.75 @-p i, 0.75 @-p i / 3,0.75 @ p i / 3,0.75 @ p i / 3$

\section{(1) 18}

$0.75 @-p i, 0.75 @ p i / 3,0,0.75 @-p i / 3$

$+00$

$0.75 @-p i, \quad 0.75 @ p i / 3, \quad 0.75 @-p i, 0.75 @-p i$

\section{WN}

$0.75 @-p i, 0.75 @ p i / 3,0.75 @-p i / 3,0$

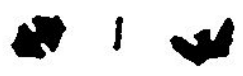

$0.75 @-p i, 0.75 @ p i / 3,0.75 @-p i / 3,0.75 @ p i / 3$

\section{$\odot \odot x$}

$0.75 @-p i, \quad 0.75 @-p i, \quad 0.75 @-p i, \quad 0.75 @-p i$

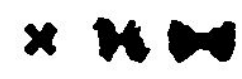

$0.75 @-p i, 0.75 \Theta-p i, \quad 0.75 \Theta-p i / 3,0$

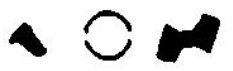

$0.75 @-p i, \quad 0.75 @-p i, \quad 0.75 @-p i / 3, \quad 0.75 @ p i / 3$

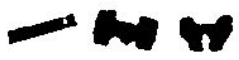

$0.75 @-p i, 0.75 @-p i, \quad 0.75 @ p i / 3,0.759-p i / 3$

() 00

$0.75 @-p i, 0.75 @-p i / 3,0,0.75 @-p i$

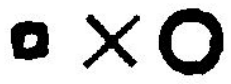

0.75@-pi, 0.75@-pi/3, 0.75@-pi, 0

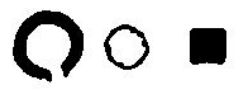

$0.75 @-p i, 0.75 @-p i / 3,0.75 @-p i, 0.75 @ p i / 3$

\section{0}

$0.75 @-p i, 0.75 @-p i / 3,0.75 @-p i / 3,0.75 @-p i / 3$

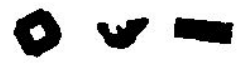

$0.75 @-p i, 0.75 @-p i / 3,0.75 @ p i / 3,0.75 @-p i$

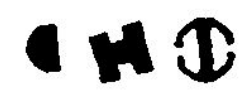

$0.75 @-p i, 0.75 \Theta p i / 3,0,0$

\section{0}

$0.75 @-p i, 0.75 @ p i / 3,0,0.75 @ p i / 3$

\section{(1) 8}

$0.75 @-p i, 0.75 \Theta p i / 3, \quad 0.75 @-p i, 0.75 @-p i / 3$

\section{$O+()$}

$0.75 @-p i, \quad 0.75 @ p i / 3, \quad 0.75 @-p i / 3,0.75 @-p i$

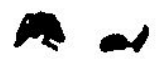

$0.75 @-p i, 0.75 @ p i / 3,0.75 @ p i / 3,0$
OX+

$0.75 @-p i, \quad 0.75 @-p i, \quad 0.75 @-p i, \quad 0.75 @-p i / 3$

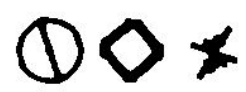

$0.75 @-p i, \quad 0.75 @-p i, \quad 0.75 @-p i / 3,0.75 @-p i$

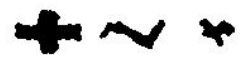

$0.75 @-p i, \quad 0.75 @-p i, \quad 0.75 @ p i / 3,0$

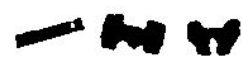

$0.75 @-p i, \quad 0.75 @-p i, \quad 0.75 @ p i / 3, \quad 0.759 p i / 3$

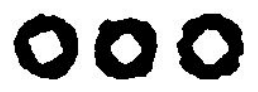

$0.75 @-p i, 0.75 @-p i / 3,0,0.75 @-p i / 3$

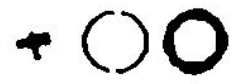

$0.75 @-p i, \quad 0.75 @-p i / 3,0.75 \Theta-p i, \quad 0.75 \Theta-p i$

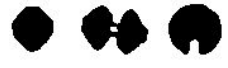

$0.75 @-p i, 0.75 @-p i / 3,0.75 @-p i / 3,0$

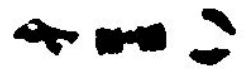

$0.75 @-p i, \quad 0.75 @-p i / 3,0.75 @-p i / 3,0.75 @ p i / 3$

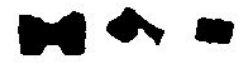

$0.75 @-p i, 0.75 @-p i / 3,0.75 @ p i / 3,0.75 @-p i / 3$

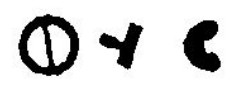

$0.75 @-p i, \quad 0.75 @ p i / 3,0,0.75 @-p i$

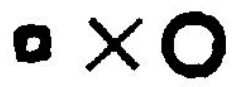

$0.75 @-p i, 0.75 @ p i / 3,0.75 @-p i, 0$

\section{\& \& $*$}

0.75@-pi, 0.75@pi/3, $0.75 @-p i, \quad 0.759 p i / 3$

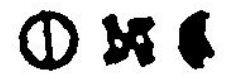

$0.75 @-p i, 0.75 @ p i / 3,0.75 @-p i / 3,0.75 @-p i / 3$

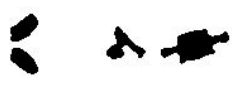

$0.75 @-p i, \quad 0.75 @ p i / 3,0.75 @ p i / 3,0.75 @-p i$ 


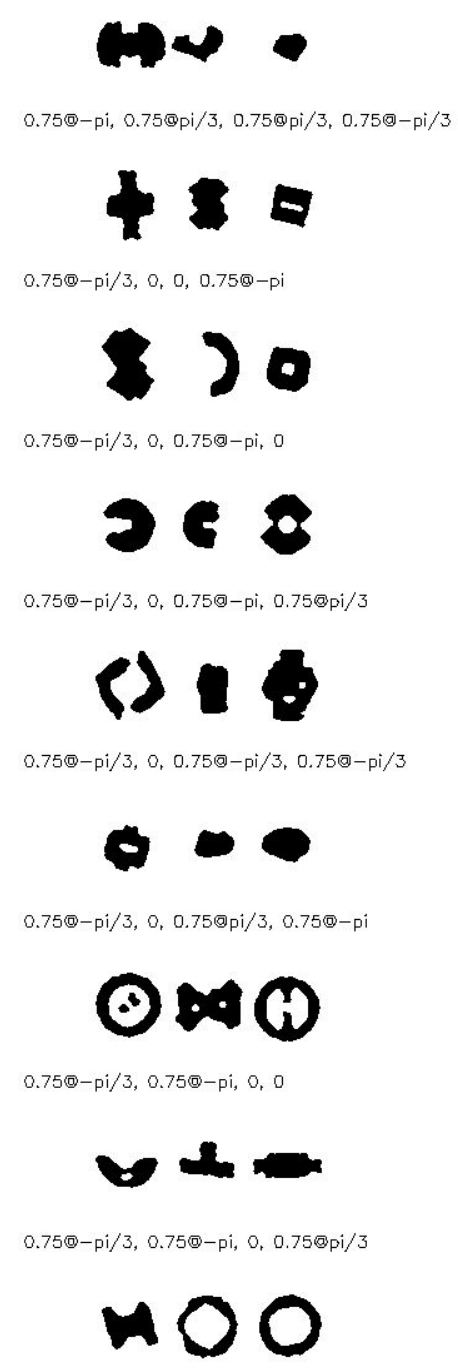

$0.75 @-p i / 3,0.75 @-p i, \quad 0.75 @-p i, \quad 0.75 @-p i / 3$

\section{$\odot \circ x$}

$0.75 @-p i / 3,0.75 @-p i, 0.75 @-p i / 3,0.75 @-p i$

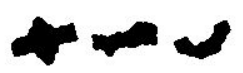

$0.75 @-p i / 3,0.75 @-p i, 0.75 @ p i / 3,0$

\section{$1=$}

$0.75 @-p i / 3,0.75 @-p i, 0.75 @ p i / 3,0.75 @ p i / 3$

\section{0}

$0.75 @-p i / 3,0.75 @-p i / 3,0,0.75 @-p i / 3$

\section{$\Leftrightarrow 2 \neq$}

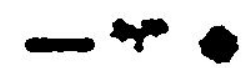

$0.75 @-p i, 0.75 @ p i / 3,0.75 @ p i / 3,0.75 @ p i / 3$

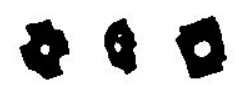

$0.75 @-p i / 3,0,0,0.75 @-p i / 3$

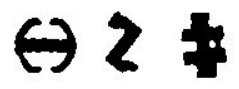

$0.75 @-p i / 3,0,0.75 @-p i, 0.75 @-p i$

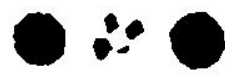

$0.75 @-p i / 3,0,0.75 @-p i / 3,0$

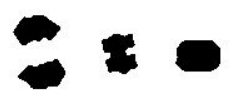

$0.75 @-p i / 3,0,0.75 @-p i / 3,0.75 @ p i / 3$

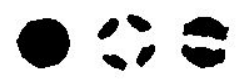

$0.75 @-p i / 3,0,0.75 @ p i / 3,0.75 @-p i / 3$

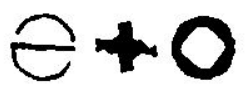

$0.75 @-p i / 3,0.75 @-p i, \quad 0, \quad 0.75 @-p i$

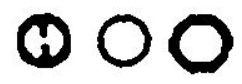

$0.75 @-p i / 3,0.75 @-p i, 0.75 @-p i, 0$

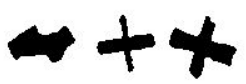

$0.75 @-p i / 3,0.75 @-p i, \quad 0.75 @-p i, 0.75 @ p i / 3$

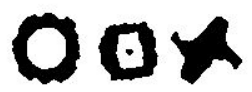

$0.75 @-p i / 3,0.75 @-p i, 0.75 \Theta-p i / 3,0.75 \Theta-p i / 3$

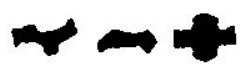

$0.75 @-p i / 3,0.75 @-p i, 0.75 @ p i / 3,0.75 @-p i$

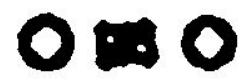

$0.75 @-p i / 3,0.75 @-p i / 3,0,0$

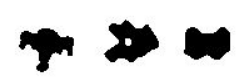

$0.75 @-p i / 3,0.75 @-p i / 3,0,0.75 @ p i / 3$

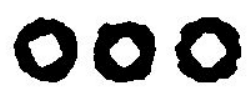

$0.750-p i / 3,0,0,0$

$$
\rightarrow 0
$$

$0.75 @-p i / 3,0,0,0.75 @ p i / 3$

\section{I.3}

$0.75 @-p i / 3,0,0.75 @-p i, 0.75 @-p i / 3$

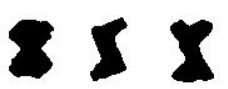

$0.75 @-p i / 3,0,0.75 @-p i / 3,0.75 @-p i$

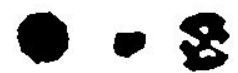

$0.75 @-p i / 3,0,0.75 @ p i / 3,0$

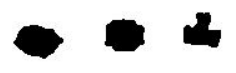

$0.75 @-p i / 3,0,0.75 @ p i / 3,0.75 @ p i / 3$

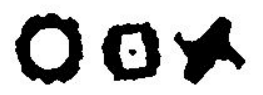

$0.75 @-p i / 3,0.75 @-p i, \quad 0,0.75 @-p i / 3$

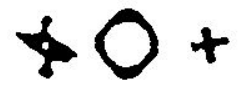

$0.75 @-p i / 3,0.75 @-p i, 0.75 @-p i, 0.75 @-p i$

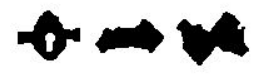

$0.75 @-p i / 3,0.75 @-p i, 0.75 @-p i / 3,0$

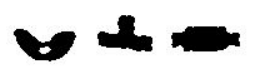

$0.75 @-\mathrm{pi} / 3,0.75 @-\mathrm{pi}, 0.75 \Theta-\mathrm{pi} / 3,0.75 \Theta \mathrm{pi} / 3$

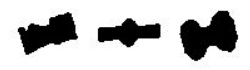

$0.75 @-p i / 3,0.75 @-p i, 0.75 @ p i / 3,0.75 @-p i / 3$

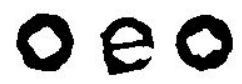

$0.75 @-p i / 3,0.75 @-p i / 3,0,0.75 @-p i$

\section{3}

$0.75 @-p i / 3,0.75 @-p i / 3,0.75 @-p i, 0$

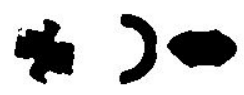




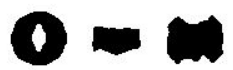
$\theta \theta \theta$
000

$0.75 @-p i / 3,0.75 @-p i / 3,0.75 @-p i / 3,0$

$0.75 @-p i / 3,0.75 @-p i / 3,0.75 @-p i / 3,0.75 @-p i \quad 0.75 @-p i / 3,0.75 @-p i / 3,0.75 @-p i / 3,0.75 @-p i /$
ras
$x=$
00

$0.75 @-p i / 3,0.75 @-p i / 3,0.75 @-p i / 3,0.75 @ p i / 3 \quad 0.75 @-p i / 3,0.75 @-p i / 3,0.75 @ p i / 3,0$

$0.75 @-p i / 3,0.75 @-p i / 3,0.75 @ p i / 3,0.75 @-p i$
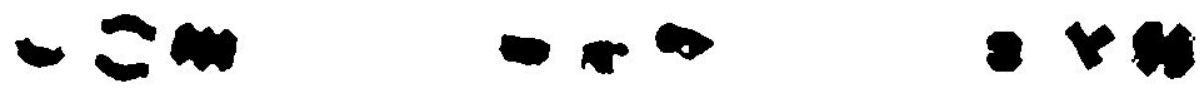

$0.75 @-p i / 3,0.75 @-p i / 3,0.75 @ p i / 3,0.75 @-p i / 3 \quad 0.75 @-p i / 3,0.75 @-p i / 3,0.75 @ p i / 3,0.75 @ p i / 3 \quad 0.75 @-p i / 3,0.75 @ p i / 3,0,0$

$+\$$

$0.75 @-p i / 3,0.75 @ p i / 3,0,0.75 @-p i$

30

$0.75 @-\mathrm{pi} / 3,0.75 @ \mathrm{pi} / 3,0.75 @-\mathrm{pi}, 0$

\section{$3 \%$}

$0.75 @-\mathrm{pi} / 3,0.75 @ \mathrm{pi} / 3,0.75 @-\mathrm{pi}, 0.75 @ \mathrm{pi} / 3$

\section{1}

$0.75 @-p i / 3,0.75 @ p i / 3,0.75 @-p i / 3,0.75 @-p i / 3 \quad 0.75 @-p i / 3,0.75 @ p i / 3,0.75 @-p i / 3,0.75 @ p i / 3$

$\wedge \wedge$

$0.75 @-\mathrm{pi} / 3,0.75 @ \mathrm{pi} / 3,0.75 @ \mathrm{pi} / 3,0.75 @-\mathrm{pi}$

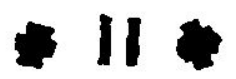

$0.75 @ \mathrm{pi} / 3,0,0,0$

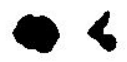

$0.75 @ \mathrm{pi} / 3,0,0,0.75 @ \mathrm{pi} / 3$

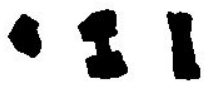

$0.75 \Theta \mathrm{pi} / 3,0,0.75 \Theta-\mathrm{pi}, 0.75 \Theta-\mathrm{pi} / 3$

() 18

$0.75 @ \mathrm{pi} / 3,0,0.75 \Theta-\mathrm{pi} / 3,0.75 \Theta-\mathrm{pi}$

$$
0-1-0
$$

$0.75 @ \mathrm{pi} / 3,0,0.75 @ \mathrm{pi} / 3,0$

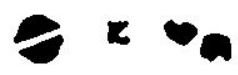

$0.75 @ \mathrm{pi} / 3,0,0.75 @ \mathrm{pi} / 3,0.75 @ \mathrm{pi} / 3$

\section{1}

$0.75 @-p i / 3,0.75 @ p i / 3,0,0.75 @-p i / 3$

$\Leftrightarrow 2 \neq$

$0.75 @-p i / 3,0.75 @ p i / 3,0.75 @-p i, 0.75 @-p i$

\section{$\infty$ (i)}

$0.75 @-p i / 3,0.75 @ p i / 3,0.75 @-p i / 3,0$ $\bullet+\bullet$

$0.75 @-\mathrm{pi} / 3,0.75 @ \mathrm{pi} / 3,0,0.75 @ \mathrm{pi} / 3$

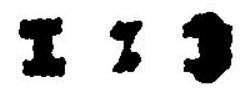

$0.75 @-p i / 3,0.75 @ p i / 3,0.75 @-p i, 0.75 @-p i / 3$

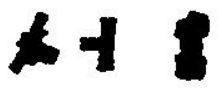

$0.75 @-p i / 3,0.75 @ p i / 3,0.75 @-p i / 3,0.75 @-p i$

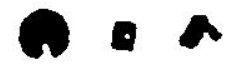

$0.75 @-p i / 3,0.75 @ p i / 3,0.75 @ p i / 3,0$

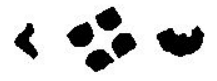

$0.75 @-p i / 3,0.75 @ p i / 3,0.75 @ p i / 3,0.75 @-p i / 3$

$0.75 @-\mathrm{pi} / 3,0.75 @ \mathrm{pi} / 3,0.75 @ \mathrm{pi} / 3,0.75 @ \mathrm{pi} / 3$

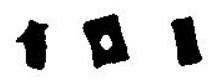

$0.75 @ \mathrm{pi} / 3,0,0,0.75 @-\mathrm{pi}$

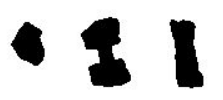

$0.75 @ \mathrm{pi} / 3,0,0.75 @-\mathrm{pi}, 0$

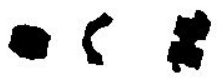

$0.75 @ \mathrm{pi} / 3,0,0.75 @-\mathrm{pi}, 0.75 @ \mathrm{pi} / 3$

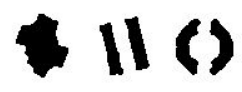

$0.75 @ \mathrm{pi} / 3,0,0.75 @-\mathrm{pi} / 3,0.75 \Theta-\mathrm{pi} / 3$

$$
0-1-0
$$

$0.75 @ \mathrm{pi} / 3,0,0.75 @ \mathrm{pi} / 3,0.75 @-\mathrm{pi}$

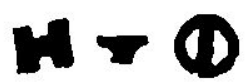

0.75@pi/3, 0.75@-pi, 0, o
$0.75 @ \mathrm{pi} / 3,0,0,0.75 @-\mathrm{pi} / 3$

$$
\text { I' }
$$

$0.75 @ \mathrm{pi} / 3,0,0.75 \Theta-\mathrm{pi}, 0.75 \Theta-\mathrm{pi}$

\section{unv}

$0.75 @ p i / 3,0,0.75 @-p i / 3,0$

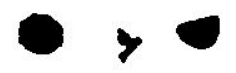

$0.75 @ p i / 3,0,0.75 \Theta-p i / 3,0.75 \Theta p i / 3$

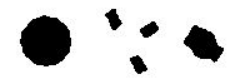

$0.75 @ \mathrm{pi} / 3,0,0.75 @ \mathrm{pi} / 3,0.75 @-\mathrm{pi} / 3$

\section{$\times>$}


OO II

$0.75 @ p i / 3,0.75 @-p i, 0,0.75 @-p i / 3$

\section{\$?}

$0.75 @ p i / 3,0.75 @-p i, 0.75 @-p i, 0.759-p i$

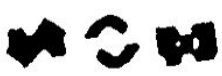

$0.75 @ p i / 3,0.75 @-p i, 0.75 @-p i / 3,0$

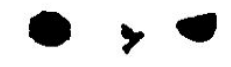

$0.75 @ p i / 3,0.75 @-p i, 0.75 \Theta-p i / 3,0.75 @ p i / 3$

42

$0.75 @ p i / 3,0.75 @-p i, 0.75 @ p i / 3,0.75 @-p i / 3$

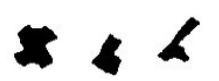

$0.75 @ p i / 3,0.75 @-p i / 3, \quad 0,0.75 \oplus-p i$

\section{1}

$0.75 @ p i / 3,0.75 @-p i / 3,0.75 @-p i, 0$

\section{$12 \cdot$}

$0.75 \oplus p i / 3,0.75 @-p i / 3,0.75 @-p i, 0.75 @ p i / 3$

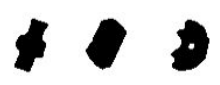

$0.75 @ \mathrm{pi} / 3,0.75 @-\mathrm{pi} / 3,0.75 @-\mathrm{pi} / 3,0.75 @-\mathrm{pi} / 30.75 @ \mathrm{pi} / 3,0.75 @-\mathrm{pi} / 3,0.75 @-\mathrm{pi} / 3,0.75 @ \mathrm{pi} / 3$

- $\bullet$

$0.75 @ p i / 3,0.75 @-p i / 3,0.75 @ p i / 3,0.75 @-p i$

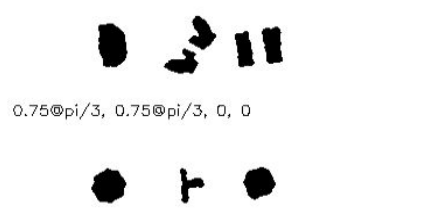

$0.75 @ \mathrm{pi} / 3,0.75 @ \mathrm{pi} / 3,0,0.75 @ \mathrm{pi} / 3$

$\$$

$0.75 @ p i / 3,0.75 @ p i / 3,0.750-p i, 0.750-p i / 3$

\section{1}

$0.75 @ p i / 3,0.75 @ p i / 3,0.75 @-p i / 3,0.75 @-p i$

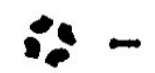

$0.75 @ \mathrm{pi} / 3,0.75 @ \mathrm{pi} / 3,0.75 @ \mathrm{pi} / 3,0$

\section{* 8}

$0.75 @ \mathrm{pi} / 3,0.75 @ \mathrm{pi} / 3,0.75 @ \mathrm{pi} / 3,0.750 \mathrm{pi} / 3$
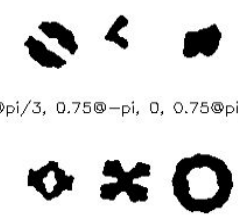

$0.75 @ p i / 3,0.75 @-p i, \quad 0.75 @-p i, 0.75 @-p i / 3$

$$
\text { () } 8
$$

$0.75 \Theta \mathrm{pi} / 3,0.75 \Theta-\mathrm{pi}, 0.75 \Theta-\mathrm{pi} / 3,0.75 \Theta-\mathrm{pi}$

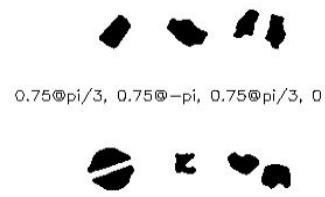

$0.75 @ p i / 3,0.75 @-p i, 0.75 @ p i / 3,0.75 @ p i / 3$

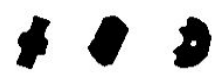

$0.75 @ p i / 3,0.75 @-p i / 3, \quad 0,0.75 @-p i / 3$

\section{II}

$0.75 @ \mathrm{pi} / 3,0.75 @-\mathrm{pi} / 3,0.75 @-\mathrm{pi}, 0.75 @-\mathrm{pi}$

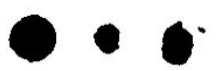

$0.75 @ p i / 3,0.75 @-p i / 3,0.75 @-p i / 3,0$

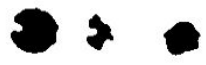

$0.75 @ \mathrm{pi} / 3,0.75 @-\mathrm{pi}, 0,0.75 @ \mathrm{pi} / 3$

$0 \times 0$

$0.75 @ p i / 3,0.75 @-p i, 0.75 @-p i, 0$

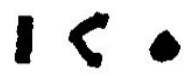

$0.75 @ p i / 3, \quad 0.75 @-p i, \quad 0.75 @-p i, 0.759 \mathrm{pi} / 3$

OO II

$0.75 @ p i / 3,0.75 @-p i, 0.75 \Theta-p i / 3,0.75 @-p i / 3$

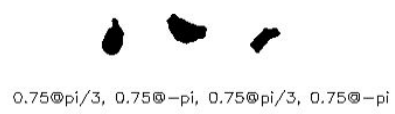

$0>0$

$0.75 @ \mathrm{pi} / 3,0.75 @-\mathrm{pi} / 3,0,0$

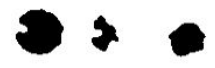

$0.75 @ \mathrm{pi} / 3,0.75 @-\mathrm{pi} / 3,0,0.75 @ \mathrm{pi} / 3$

\section{1}

$0.75 @ p i / 3,0.75 @-p i / 3,0.75 @-p i, 0.75 @-p i / 3$

\section{I}

$0.75 @ p i / 3,0.75 @-p i / 3,0.75 @-p i / 3,0.75 \circledast-p i$

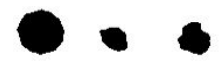

$0.75 @ \mathrm{pi} / 3,0.75 @-\mathrm{pi} / 3,0.759 \mathrm{pi} / 3,0$

$$
1
$$

$$
\geqslant \times \bullet_{0}
$$

$0.75 @ p i / 3,0.75 @-p i / 3,0.75 @ p i / 3,0.75 @-p i / 3$

$0.75 @ p i / 3,0.75 @-p i / 3,0.75 @ p i / 3,0.75 @ p i / 3$
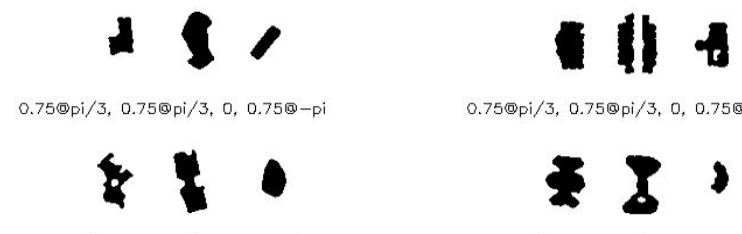

$0.75 @ \mathrm{pi} / 3,0.75 @ \mathrm{pi} / 3,0,0.75 @-\mathrm{pi} / 3$

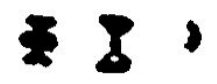

$0.75 @ p i / 3,0.75 @ p i / 3,0.75 @-p i, 0$

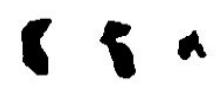

11.

$0.75 @ \mathrm{pi} / 3,0.75 @ \mathrm{pi} / 3,0.75 @-\mathrm{pi}, 0.75 @ \mathrm{pi} / 3$

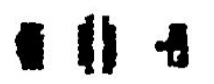

- $r$

$0.75 @ p i / 3,0.75 @ p i / 3,0.75 @-p i / 3,0.75 @-p i / 3 \quad 0.75 @ p i / 3,0.75 @ p i / 3,0.75 @-p i / 3,0.75 @ p i / 3$

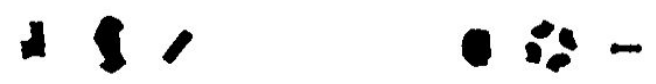

$0.75 @ \mathrm{pi} / 3,0.75 @ \mathrm{pi} / 3,0.75 @ \mathrm{pi} / 3,0.75 @-\mathrm{pi}$

$0.75 @ \mathrm{pi} / 3,0.75 @ \mathrm{pi} / 3,0.75 @ \mathrm{pi} / 3,0.759-\mathrm{pi} / 3$ 
Figure. S4. The detailed structures of all the 256 unique supercells for the space-polarizationwavelength hologram. This figure spans multiple pages. Each supercell consists of three layers, and the structure of each layer (from the top to the bottom) is presented sequentially. The four numbers below each supercell are their target responses at $x$ and $y$-polarized incidence at $562 \mathrm{~nm}$ $\left(\lambda_{1}\right)$ and $659 \mathrm{~nm}\left(\lambda_{2}\right)$, denoted as $E_{\lambda_{1} x}, E_{\lambda_{1} y}, E_{\lambda_{2} x}, E_{\lambda_{2} y}$.

\section{High-resolution output images of the space-polarization-wavelength multiplexed hologram}

In the main text, Figure 4(d) shows the simulated output of the space-polarization-wavelength multiplexed hologram. Here we present the high-resolution images of all 36 simulated output images in Figure S5.

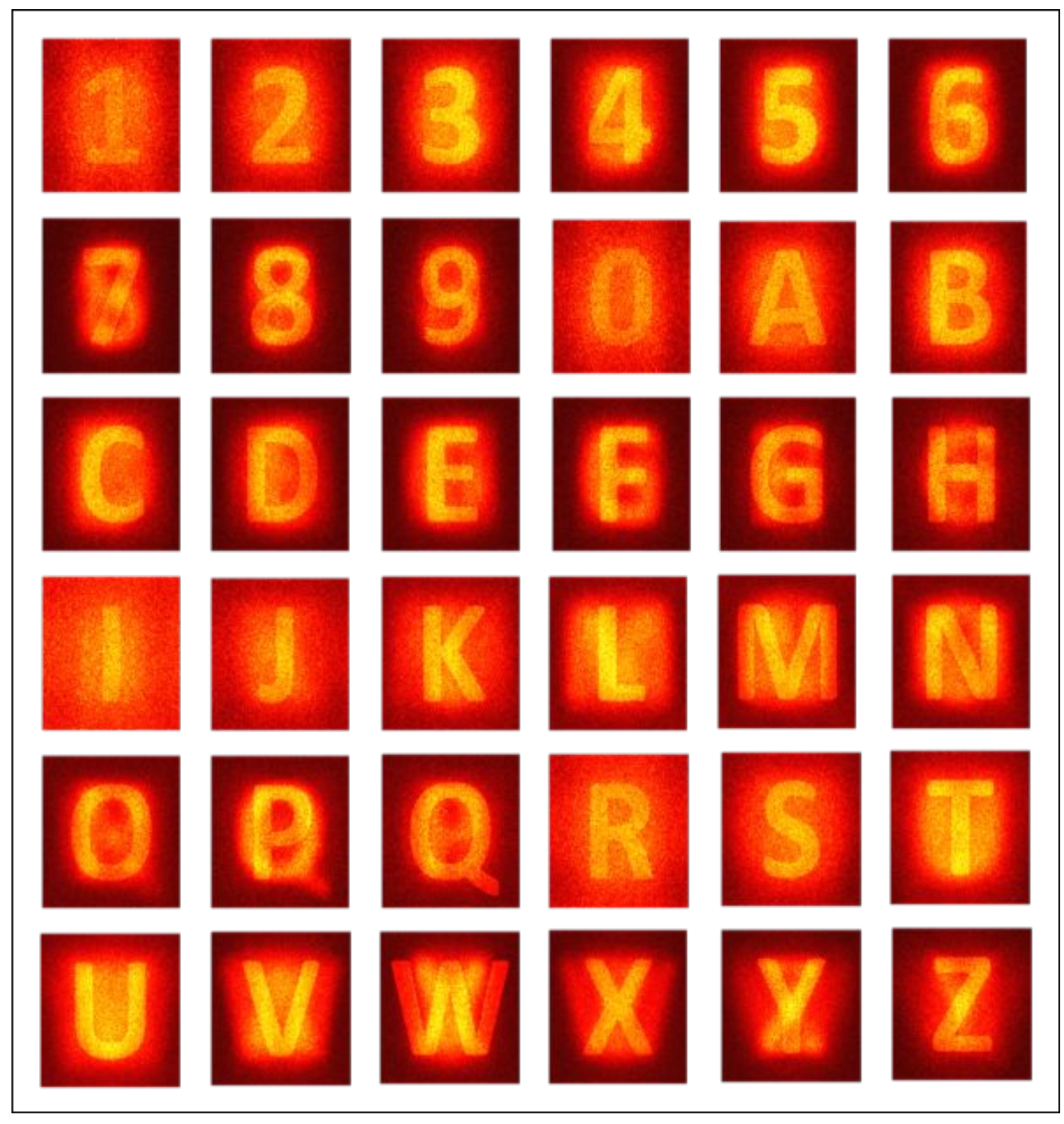


Figure S5. High-resolution simulated images of the space-polarization-wavelength multiplexed hologram.

\section{Discussion on the hologram performance and capacity}

In the maintext, as shown in Figure 4d, some of the projected images are not perfect in terms of the sharpness and legibility, which mainly result from crosstalk with other images. There are two major causes of the crosstalk. First, as the projection distance increases, the light propagation will exhibit more of the nature of Fraunhofer diffraction than Fresnel diffraction. As a result, the projected image will mostly represent the Fourier transformation of the input hologram regardless of the distance, rather than the convolution between the hologram and the distance-related transfer function. This is consistent with the observation from Figure $4 \mathrm{~d}$ that the blurring or crosstalk of the images becomes more noticeable at longer distances. This dilemma can be settled by adding a Fresnel plate before the hologram to convert all the transformation into the near-field regime. ${ }^{9}$ Second, if a projected image is located between two similar images (e.g. "7" between "6" and "8", "P" between "O" and "Q"), it will suffer more from crosstalk than regular cases. Considering the crosstalk stemming from the nonorthogonality between the projected matrices of the images, the image (e.g., "P") sandwiched between two very similar images (e.g., "O" and "Q") will be hardly orthogonal to both, and the crosstalk subsequently arises. To mitigate this shortcoming, the phase randomization process in Figure 4a can be replaced by a Gram-Schmidt process to guarantee the orthogonality of the projected matrices of the phase-added images. As for the scalability, the 256 unique supercells can be readily adapted to design any space-polarization-wavelength multiplexed holograms for the polarization states and operating wavelengths specified before. Additional examples are presented in the Supporting Information. It is worth noting that our method can be extended to the design of holograms at more operating wavelengths and image positions. Adding one wavelength with both polarizations will increase the number of unique supercells by 16 times.

\section{Additional examples of the space-polarization-wavelength multiplexed hologram}

Here in Figure S6 we present an additional example of the space-polarization-wavelength multiplexed hologram. The hologram works for $x$ - and $y$-polarizations at $659 \mathrm{~nm}$ and $562 \mathrm{~nm}$, and the projected images are at 100, 200 and $300 \mu \mathrm{m}$ from the hologram plane. The hologram consists of 1000 by 1400 supercells, which are selected from the pool of the 256 unique supercells. The projected images are 0 to 9 , with the plus and the equality signs, for a total of 12 images. 


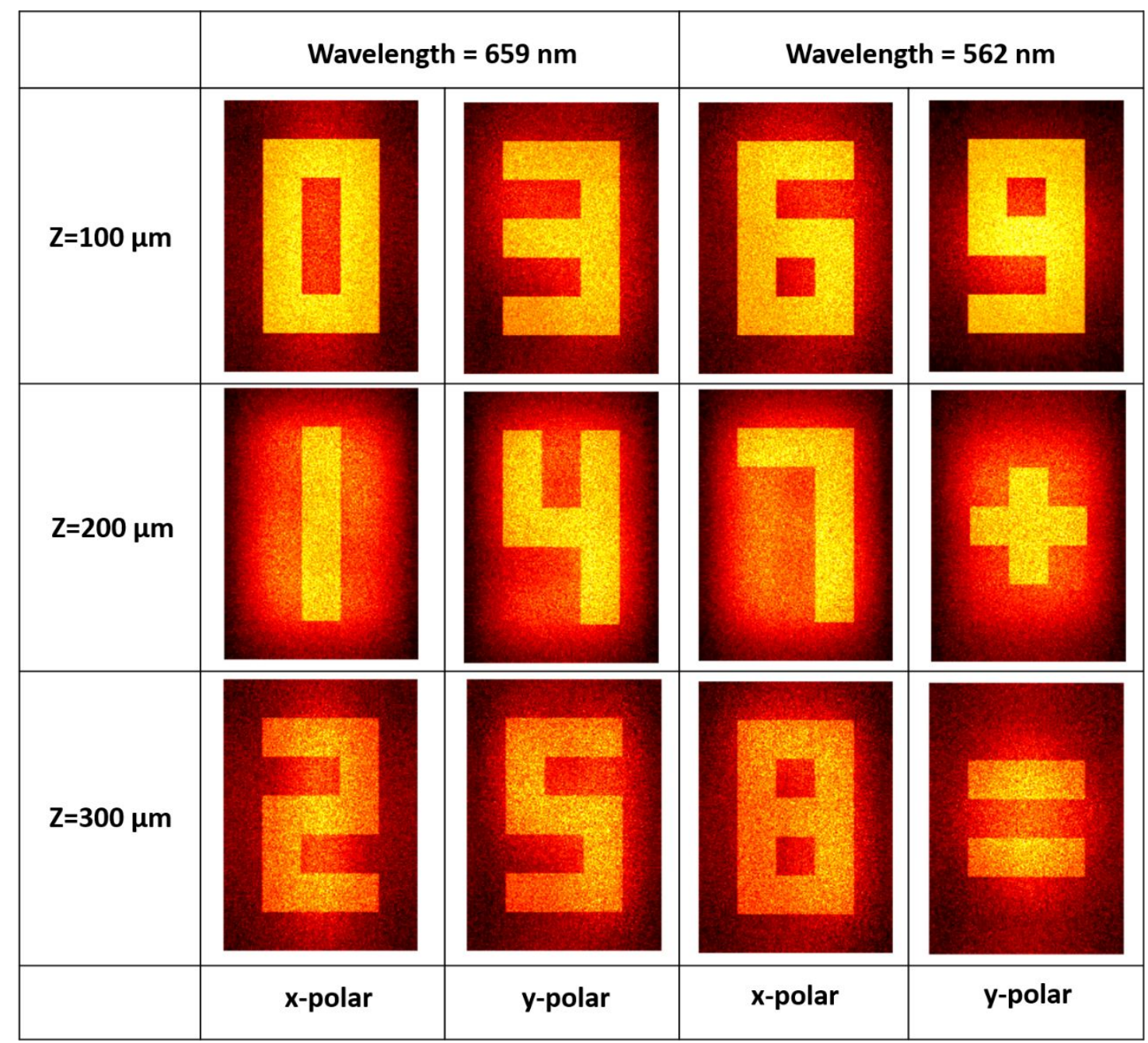

Figure S6. An additional example of the space-polarization-wavelength multiplexed hologram. The hologram can project 12 images, including the numbers 0 to 9, a plus sign, and an equality sign. The hologram works for $x$ - and $y$-polarizations at $659 \mathrm{~nm}$ and $562 \mathrm{~nm}$, and the projected images are 100, 200 and $300 \mu \mathrm{m}$ from the hologram plane. 


\section{References}

1) Stanley, K. O. Compositional Pattern Producing Networks: A Novel Abstraction of Development. Genet. Program. Evolvable Mach. 2007, 8, 131-162.

2) Goodfellow, I.; Pouget-Abadie, J.; Mirza, M.; Xu, B.; Warde-Farley, D.; Ozair, S.; Courville, A.; Bengio, Y. Generative Adversarial Nets, Advances in Neural Information Processing Systems, Montreal, Canada, December, 2014, pp 2672-2680.

3) LeCun, Y.; Bottou, L.; Bengio, Y.; Haffner, P. Gradient-Based Learning Applied to Document Recognition. Proc. IEEE 1998, 86, 2278-2324.

4) He, K.; Zhang, X.; Ren, S.; Sun, J. Deep Residual Learning for Image Recognition, Proceedings of the IEEE Conference on Computer Vision and Pattern Recognition, Las Vegas, USA, June, 2016, pp 770778.

5) Ranjbar, A.; Grbic, A. Analysis and Synthesis of Cascaded Metasurfaces Using Wave Matrices. Phys. Rev. B 2017, 95, 205114.

6) Liu, Z.; Zhu, Z.; Cai, W. Topological Encoding Method for Data-Driven Photonics Inverse Design. Opt. Express 2020, 28, 4825-4835.

7) Liu, Z.; Zhu, D.; Lee, K. T.; Kim, A. S.; Raju, L.; Cai, W. Compounding Meta-Atoms into Metamolecules with Hybrid Artificial Intelligence Techniques. Adv. Mater. 2020, 32, 1904790.

8) Silva, A.; Monticone, F.; Castaldi, G.; Galdi, V.; Alù, A.; Engheta, N. Performing Mathematical Operations with Metamaterials. Science 2014, 343, 160-163.

9) Makey, G.; Yavuz, Ö.; Kesim, D. K.; Turnal1, A.; Elahi, P.; Ilday, S.; Tokel, O.; Ilday, F. Ö. Breaking Crosstalk Limits to Dynamic Holography Using Orthogonality of High-Dimensional Random Vectors. Nat. Photonics 2019, 13, 251-256. 